\title{
Publisher's Note: Molecular oxygen observed by direct photoproduction from carbon dioxide [Phys. Rev. A 95, 011404(R) (2017)]
}

\author{
Seyedreza Larimian, Sonia Erattupuzha, Sebastian Mai, Philipp Marquetand, Leticia González, Andrius Baltuška, \\ Markus Kitzler, and Xinhua Xie (谢新华) \\ (Received 13 February 2017; published 22 February 2017)
}

DOI: 10.1103/PhysRevA.95.029903

This paper was published online on 27 January 2017 with an incorrect label in Fig. 1(b). The figure label has been corrected as of 10 February 2017. The figure label is incorrect in the printed version of the journal; therefore for the benefit of the print readership, the figure is replicated below.

(a)

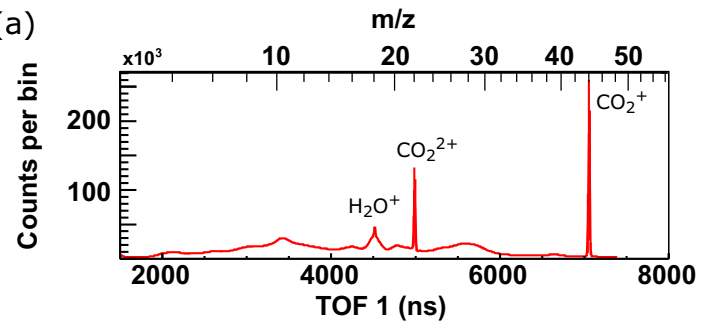

(b)

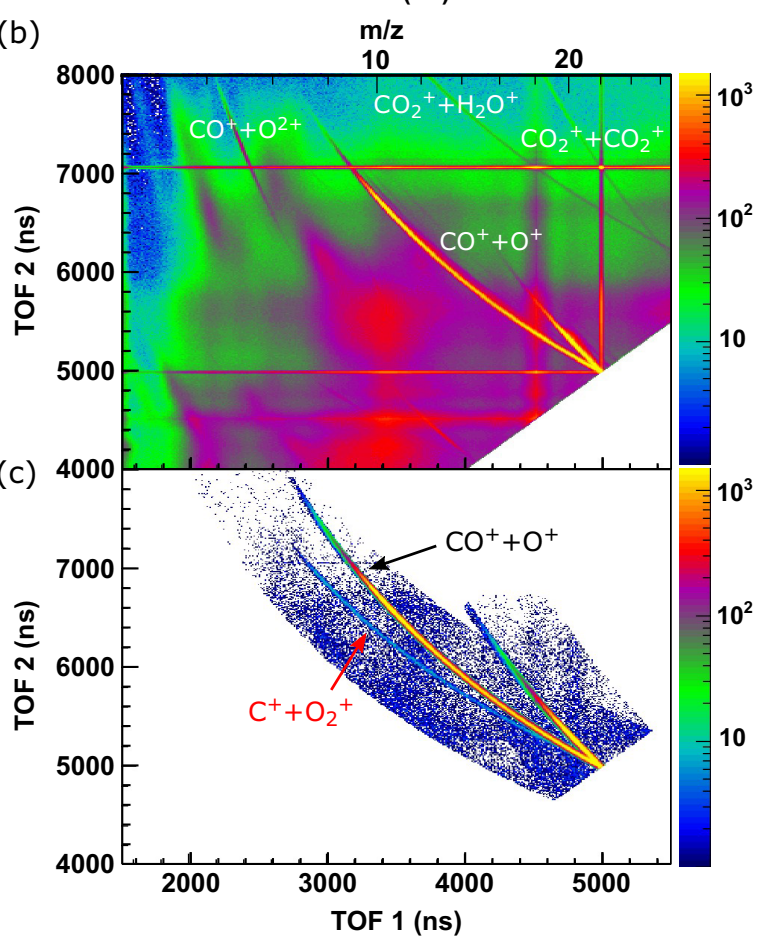

(d)

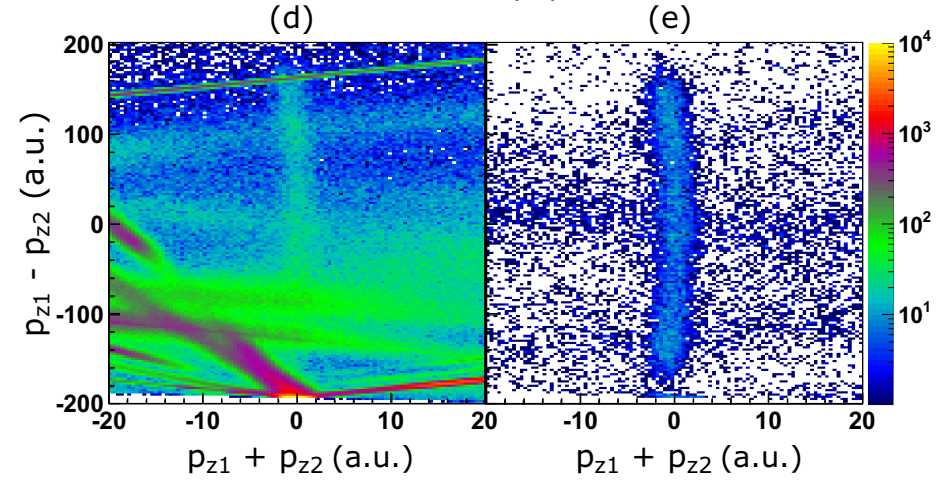

FIG. 1. (a) Measured time-of-flight spectrum of photoions. (b) and (c) present measured photoion-photoion coincidence distributions without and with selection conditions for the two fragmentation channels, respectively. (d) and (e) illustrate momentum correlation distributions for the $\mathrm{C}^{+}+\mathrm{O}_{2}{ }^{+}$channel along the laser polarization direction between $\mathrm{C}^{+}$and $\mathrm{O}_{2}{ }^{+}$ions before and after applying the coincidence selection, respectively. The two-body coincidence selection conditions include momentum conservation conditions of $\left|p_{x 1}+p_{x 2}\right|<2$ a.u. and $\left|p_{y 1}+p_{y 2}\right|<5$ a.u., and a further condition of $\sqrt{p_{x 1}^{2}+p_{y 1}^{2}}>30$ a.u. for the $\mathrm{C}^{+}+\mathrm{O}_{2}{ }^{+}$ channel to get rid of overlapping with other signals, where the $x$ and $y$ directions are defined along the gas jet and the laser propagation direction, and the indexes 1 and 2 indicate the first and the second considering particles, respectively. These coincidence conditions, together with the momentum conservation condition along the $z$ direction $\left(\left|p_{z 1}+p_{z 2}\right|<3\right.$ a.u.), are applied in the data analysis for further studies. 